\title{
Myocardial deformation in patients with a single left ventricle using 2D cardiovascular magnetic resonance feature tracking: a case-control study
}

\author{
Fabian Strodka ${ }^{1} \cdot J_{a n a}$ Logoteta $^{2} \cdot$ Roman Schuwerk $^{1} \cdot$ Mona Salehi Ravesh $^{3} \cdot$ Dominik Daniel Gabbert $^{1}$. \\ Anselm Sebastian Uebing ${ }^{1} \cdot$ Sylvia Krupickova ${ }^{4} \cdot$ Inga Voges $^{1}$ (I)
}

Received: 15 February 2021 / Accepted: 23 March 2021 / Published online: 31 March 2021

(c) The Author(s) 2021

\begin{abstract}
Ventricular dysfunction is a well-known complication in single ventricle patients in Fontan circulation. As studies exclusively examining patients with a single left ventricle (SLV) are sparse, we assessed left ventricular (LV) function in SLV patients by using 2D-cardiovascular magnetic resonance (CMR) feature tracking (2D-CMR-FT) and 2D-speckle tracking echocardiography (2D-STE). 54 SLV patients (11.4, 3.1-38.1 years) and 35 age-matched controls (12.3, 6.3-25.8 years) were included. LV global longitudinal, circumferential and radial strain (GLS, GCS, GRS) and strain rate (GLSR, GCSR, GRSR) were measured using 2D-CMR-FT. LV volumes, ejection fraction (LVEF) and mass were determined from short axis images. 2D-STE was applied in patients to measure peak systolic GLS and GLSR. In a subgroup analysis, we compared double inlet left ventricle (DILV) with tricuspid atresia (TA) patients. The population consisted of 19 DILV patients, 24 TA patients and 11 patients with diverse diagnoses. 52 patients were in NYHA class I and 2 patients were in class II. Most SLV patients had a normal systolic function but median LVEF in patients was lower compared to controls $(55.6 \%$ vs. 61.2\%, $\mathrm{p}=0.0001)$. 2D-CMR-FT demonstrated reduced GLS, GCS and GCSR values in patients compared to controls. LVEF correlated with GS values in patients $(\mathrm{p}<0.05)$. There was no significant difference between GLS values from 2D-CMR-FT and 2D-STE in the patient group. LVEF, LV volumes, GS and GSR (from 2D-CMR-FT) were not significantly different between DILV and TA patients. Although most SLV patients had a preserved EF derived by CMR, our results suggest that, LV deformation and function may behave differently in SLV patients compared to healthy subjects.
\end{abstract}

Keywords Single ventricle $\cdot$ Cardiovascular magnetic resonance $\cdot$ Feature tracking $\cdot$ Speckle tracking echocardiography

\section{Introduction}

Since the introduction of the Fontan operation, life expectancy of single ventricle (SV) patients steadily improved, and an increasing number of SV patients is reaching adolescence

Inga Voges

inga.voges@uksh.de

1 Department of Congenital Heart Disease and Paediatric Cardiology, University Hospital Schleswig-Holstein, Campus Kiel, Arnold-Heller-Str. 3, 24105 Kiel, Germany

2 Department of Paediatric Cardiology, Children's Hospital of Zurich, Zurich, Switzerland

3 Department of Radiology and Neuroradiology, University Hospital Schleswig-Holstein, Kiel, Germany

4 Royal Brompton Hospital, London, UK and adulthood [1-3]. Despite this success, we must remind ourselves that the Fontan procedure is a palliative approach that can be regarded as a bridge to cardiac transplantation with limited exceptions. SV patients with Fontan circulation are at risk for various complications including systolic and diastolic ventricular dysfunction which can significantly impact morbidity and mortality [4-7]. Registry data indicate that patients with a single left ventricle (SLV) seem to have a better freedom from late Fontan failure compared to patients with a single right ventricle (in particular hypoplastic left heart syndrome) [3].

Abnormal hemodynamics due to unfavorable volume and/ or pressure load of the $\mathrm{SV}$, as well as the stepwise surgical procedures, might be a cause for SV dysfunction and heart failure [8]. More recently associations between myocardial fibrosis and adverse SV function have been demonstrated $[9,10]$. 
Echocardiography and cardiovascular magnetic resonance (CMR) are the standard imaging modalities to evaluate SV function in patients with a Fontan circulation $[11,12]$. Beside traditional techniques, tissue tracking methods such as 2-dimensional (2D) CMR feature tracking (2D-CMR-FT) and 2D speckle tracking echocardiography (2D-STE) have gained popularity to assess global and regional myocardial deformation of the SV [13-15]. However, most studies have assessed a mixed cohort of single left (SLV) and single right ventricle (SRV) patients but only few assessed a uniform population of only SLV patients using 2D-STE $[16,17]$. Furthermore, a comparison to healthy controls was only performed in a small cohort in 2 studies [16-18].

For the present study, we hypothesized that ventricular function and myocardial deformation in patients with a SLV are impaired compared to healthy controls. We used 2D-CMR-FT and 2D-STE and analyzed myocardial deformation and ventricular function in a relatively large cohort of SLV patients and healthy controls. In addition, patients with tricuspid atresia (TA) were compared to those with a double inlet left ventricle (DILV) and 2D-CMR-FT results were compared to those from 2D-STE.

\section{Material and methods}

\section{Patient population}

This retrospective study was approved by the ethics committee of the medical faculty of the Christian-Albrechts University Kiel (No. D555/19) and included all 54 SLV patients after Fontan completion, who received a CMR examination as part of a routine clinical follow up during 2010-2019. 35 age-matched healthy controls were included for comparison. For patients who received several CMR examinations during that period, only the most recent dataset was included in our study. The comparison between 2D-CMR-FT and 2D-STE was conducted in datasets in which an echocardiography was performed within 3 months of the CMR examination. To guarantee comparability, echocardiographic studies in patients who underwent cardiovascular surgery or catheter interventions between echocardiography and CMR were excluded.

Age at examination and total cavopulmonary connection (TCPC) as well as gender, weight, height, body surface area (BSA), New York Heart Association (NYHA) functional class, transcutaneous oxygen saturation $\left(\mathrm{SpO}_{2}\right)$ and number of surgical procedures was collected from medical records. Heart rate (HR), cardiac axis and QRS duration was assessed from 12-lead electrocardiograms (ECG).

\section{Cardiovascular magnetic resonance acquisition and analysis}

CMR examinations were performed using a 3 T MRI system (Achieva TX-Series, Philips Healthcare, Best, Netherlands). In 4 older patients a 1.5 T MRI system (Achieva, Philips Healthcare, Best, Netherlands) was used. Patients were sedated using midazolam and propofol according to our clinical protocol, if necessary. Blood Pressure, HR and $\mathrm{SpO}_{2}$ levels were monitored during examination. Shortaxis, four-chamber and axial cine images were acquired using steady-state free precession or gradient echo pulse sequences. Field of view and slice thickness varied according to patient size $\left(250-400 \times 250-400 \mathrm{~mm}^{2}, 5-8 \mathrm{~mm}\right)$.

Volumetric analysis was performed using QMass (Version 8.1, Medis Medical Imaging Systems, BV, Leiden, Netherlands). Left ventricular (LV) end-diastolic and endsystolic volumes (LVEDV, LVESV) were measured from the short-axis images by manual drawing of endocardial and epicardial border at end-diastole and endocardial borders at end-systole (Fig. 1). Papillary muscles and large trabeculations were excluded from the ventricular mass and included into the ventricular volumes. The right ventricular volume was excluded as well. Left ventricular ejection fraction (LVEF), stroke volume and end-diastolic myocardial mass (LVMM) were automatically calculated by the software. Volumes and mass were indexed to BSA.

2D-CMR-FT analysis was undertaken using the dedicated software QStrain Research Edition (Version 2.0, Medis Medical Imaging Systems, BV, Leiden, Netherlands). Global longitudinal strain (GLS) and strain rate (GLSR) were measured in the four-chamber view (Fig. 1). If the examinations lacked a four-chamber-view, we used comparable axial cine images instead $(n=16)$. Global and regional circumferential strain (CS) and strain rate (CSR) as well as radial strain (RS) and strain rate (RSR) were analyzed from short axis images.

Endocardial and epicardial contours were drawn manually at end-systole, which was defined as the cardiac phase with the smallest LV cavity area. Contours were then tracked automatically by the software during the cardiac cycle. Visual inspection of the epi- and endocardial contours during the cardiac cycle was conducted to evaluate tracking quality and suggested end-diastolic contours were manually adjusted if necessary.

In the four-chamber views, the LV was automatically divided into 7 myocardial segments by the software and peak longitudinal strain and strain rate for each segment was obtained.

In the short axis, the LV was divided into 3 different levels (basal, mid-ventricular and apical) and 16 segments according to the American Heart association 

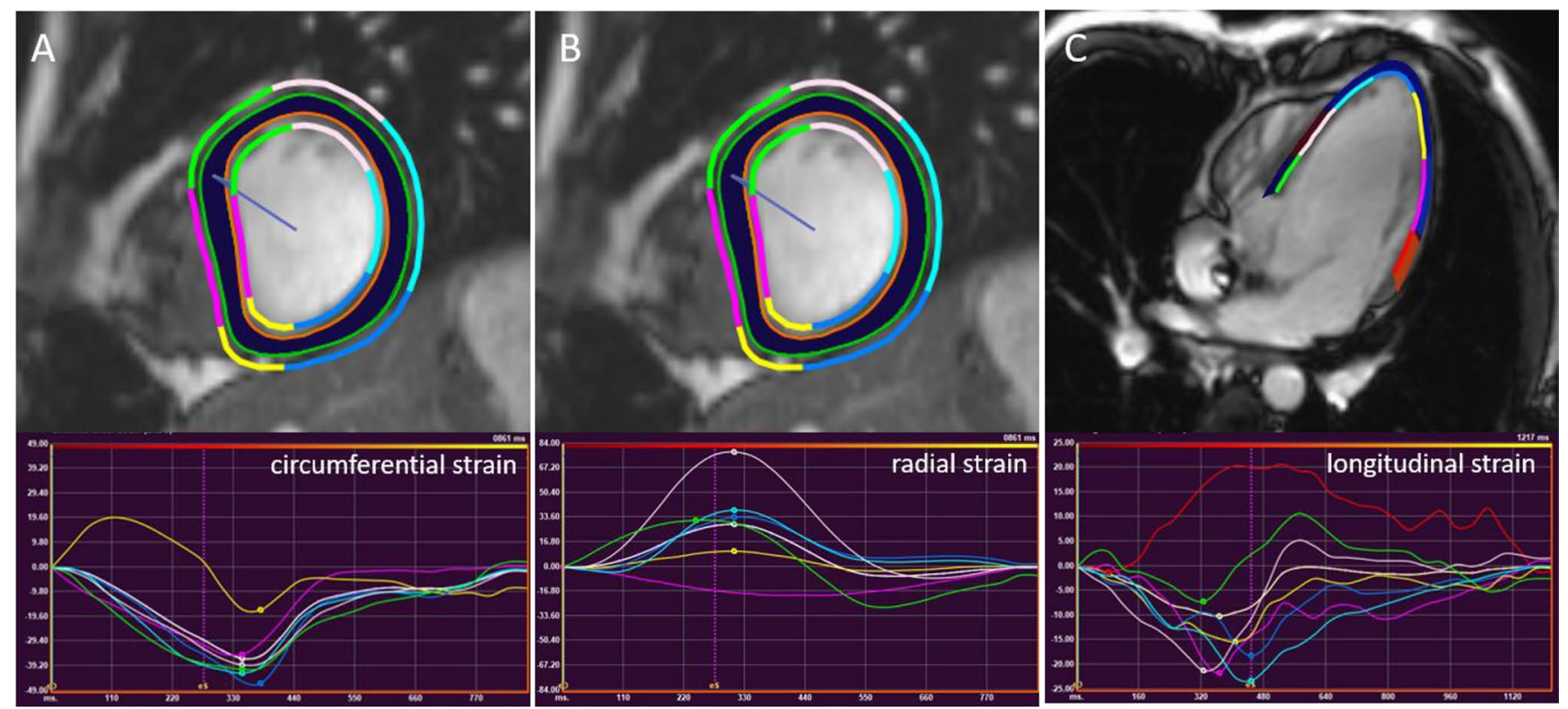

Fig. 1 Assessment of LV circumferential (a), radial (b) and longitudinal (c) strain from short axis and long axis images

16-segment-model (Fig. 1) [19]. Peak circumferential and radial strain and strain rate values were acquired for each segment.

Global strain (GS) and global strain rate (GSR) values as well as strain and strain rate values for the three ventricular levels were calculated by averaging the peak values of each segment.

\section{2-Dimensional speckle tracking echocardiography analysis}

Transthoracic echocardiography was performed using a Vivid 7 GE Dimension-System (General Electric Healthcare, Wisconsin, USA). All studies were stored digitally and were therefore available for offline analysis. The data analysis was performed using dedicated STE software (EchoPac, version 113, General Electric Healthcare, Wisconsin, USA) as previously described by our group [15].

\section{Statistics}

Statistical analysis was performed by using a dedicated software (MedCalc statistical software, version 19.5.1, software, Mariakerke, Belgium). Continuous variables were expressed either as mean \pm standard deviation if they were normally distributed, or otherwise as median with range. Normal distribution of the data was assessed using the Shapiro Wilk test. Differences between patients and controls as well as between patient subgroups were analysed using the Mann-Whitney-U test. Comparison between extracted mean values using 2D-CMR-FT and 2D-STE was performed using the paired samples t-test. Adjustments for multiple testing were performed and the significant $\mathrm{p}$-value was reduced to 0.003 . Bland-Altman plots were constructed to assess the agreement between 2D-CMR-FT and 2D-STE. Associations between variables were evaluated by the Spearman's rank method and $\mathrm{p}$ values of $<0.05$ were considered to indicate statistical significance.

\section{Results}

The patient population consisted of 24 patients with TA, 19 patients with DILV and 11 patients with diverse SLV anatomies. Characteristics of all patients and controls are presented in Table 1. Clinical characteristics for patients with TA and DILV are separately shown in Table 2 . All patients were examined after TCPC. Median age of the entire patient group was 11.4 years (range 3.1-38.1 years). All except two patients were in NYHA class I.

\section{Global and regional myocardial deformation and function}

GLS, GLSR and LV volume were determined using a 2D-CMR-FT analysis in all 54 patients. Global and regional CS, CSR, RS and RSR values were acquired in 53 patients in the short axis view. In one patient with DILV the short axis stack did not cover the entire LV.

Global systolic function derived from CMR volumetry was preserved in $52 \%(n=28)$ of SLV patients with an LVEF of $\geq 55 \%$. However, compared to the healthy controls 
Table 1 Patient characteristics and clinical data

\begin{tabular}{|c|c|c|c|}
\hline & Patients $(\mathrm{n}=56)$ & Controls $(n=35)$ & p value* \\
\hline Age at CMR examination, y & $11.4(3.1-38.1)$ & $12.3(6.3-25.8)$ & 0.28 \\
\hline Female, n $(\%)$ & $27(50)$ & $13(37)$ & - \\
\hline Body height, $\mathrm{cm}$ & $147.0(97.5-188.0)$ & $155.0(121.0-174.0)$ & 0.19 \\
\hline Body weight, $\mathrm{kg}$ & $38.7(14.3-93.0)$ & $51.0(19.0-80.0)$ & 0.05 \\
\hline BSA, $\mathrm{m}^{2}$ & $1.2(0.6-2.2)$ & $1.4(0.8-1.9)$ & 0.07 \\
\hline $\mathrm{SpO}_{2}, \%$ & $93.0(78.0-98.0)$ & & \\
\hline Age at Fontan completion, y & $2.7(1.5-26.3)$ & & \\
\hline Time since Fontan completion, y & $8.7(1.0-32.2)$ & & \\
\hline \multicolumn{4}{|l|}{ Diagnosis, $\mathrm{n}(\%)$} \\
\hline Tricuspid atresia & $24(44)$ & & \\
\hline Double inlet left ventricle, & $19(35)$ & & \\
\hline $\begin{array}{l}\text { Atrioventricular septal defect with LV } \\
\text { dominance }\end{array}$ & $3(6)$ & & \\
\hline Pulmonary atresia & $2(4)$ & & \\
\hline Other & $6(11)$ & & \\
\hline \multicolumn{4}{|l|}{ Type of Fontan, n (\%) } \\
\hline Intraatrial lateral tunnel & $43(79.6)$ & & \\
\hline Extracardiac conduit & $9(16.7)$ & & \\
\hline Fontan-Bjoerk modification & $1(1.9)$ & & \\
\hline Atriopulmonary connection & $1(1.9)$ & & \\
\hline \multicolumn{4}{|l|}{ Fenestration, n (\%) } \\
\hline Open & $27(50)$ & & \\
\hline Closed/non-fenestrated tunnel & $27(50)$ & & \\
\hline \multicolumn{4}{|l|}{ NYHA functional class, n (\%) } \\
\hline I & $52(96)$ & & \\
\hline II & $2(4)$ & & \\
\hline
\end{tabular}

$\mathrm{SpO}_{2}$ oxygen saturation, $y$ year

*Comparisons were performed using the Mann-Whitney U test

\begin{tabular}{llll}
\hline Parameter & $\begin{array}{l}\text { TA } \\
(\mathrm{n}=24)\end{array}$ & $\begin{array}{l}\text { DILV } \\
(\mathrm{n}=19)\end{array}$ & *p value \\
\hline Age at CMR examination, y & $14.1(3.1-38.1)$ & $9.4(3.9-37.0)$ & 0.22 \\
Female, $\mathrm{n}(\%)$ & $12(50)$ & $9(47)$ & - \\
Body height, cm & $158.0(97.5-188.0)$ & $135.0(99.0-180.0)$ & 0.17 \\
Body weight, kg & $48.9(14.3-92.0)$ & $39.3(16.0-93.0)$ & 0.23 \\
BSA, m ${ }^{2}$ & $1.5(0.6-2.2)$ & $1.2(0.7-2.2)$ & 0.22 \\
Age at Fontan completion, y & $2.7(2-7.7)$ & $2.5(1.5-26.3)$ & 0.18 \\
Time since Fontan completion, y & $9.3(1.0-32.2)$ & $7.6(1.3-24.6)$ & 0.34 \\
Type of Fontan, n (\%) & & & \\
Intraatrial lateral tunnel & $20(83.3)$ & $15(78.9)$ & \\
Extracardiac conduit & $3(12.5)$ & $4(21.1)$ & \\
Fontan-Bjoerk modification & $1(4.2)$ & - & \\
Fenestration, $\mathrm{n}(\%)$ & & $13(68.4)$ & \\
Open & $8(33.3)$ & $6(31.6)$ & \\
Closed/non-fenestrated tunnel & $16(66.7)$ & $18(94.7)$ & $1(5.3)$ \\
NYHA functional class, $\mathrm{n}(\%)$ & &
\end{tabular}


Table 3 Volumetric data from cardiovascular magnetic resonance imaging

\begin{tabular}{lllllll}
\hline Parameter & $\begin{array}{l}\text { SLV } \\
(\mathrm{n}=53)\end{array}$ & $\begin{array}{l}\text { Controls } \\
(\mathrm{n}=35)\end{array}$ & *p value & $\begin{array}{l}\text { TA } \\
(\mathrm{n}=24)\end{array}$ & $\begin{array}{l}\text { DILV } \\
(\mathrm{n}=18)\end{array}$ \\
\hline LVEDVi $\left(\mathrm{ml} / \mathrm{m}^{2}\right)$ & $81.3[70.8 ; 88.9]$ & $74.8[68.1 ; 84.3]$ & 0.12 & $77.7[73.7 ; 84.7]$ & $87.3[81.9 ; 101.5]$ & 0.06 \\
LVESVi $\left(\mathrm{ml} / \mathrm{m}^{2}\right)$ & $35.9[28.6 ; 44.0]$ & $28.3[25.5 ; 33.8]$ & $\mathbf{0 . 0 0 0 9}$ & $34.5[28.7 ; 43.9]$ & $40.6[35.9 ; 45.9]$ & 0.19 \\
LVSVi $\left(\mathrm{ml} / \mathrm{m}^{2}\right)$ & $43.9[40.2 ; 48.9]$ & $45.5[41.9 ; 52.1]$ & 0.44 & $43.7[38.9 ; 47.3]$ & $47.0[40.6 ; 56.9]$ & 0.14 \\
LVEF $(\%)$ & $55.6[51.4 ; 60.1]$ & $61.2[58.1 ; 64.7]$ & $\mathbf{0 . 0 0 0 1}$ & $55.1[49.0 ; 61.9]$ & $53.1[51.0 ; 58.5]$ & 0.67 \\
LVMMi $\left(\mathrm{g} / \mathrm{m}^{2}\right)$ & $49.7[43.4 ; 58.3]$ & $47.2[42.8 ; 55.6]$ & 0.41 & $48.2[40.1 ; 60.1]$ & $51.6[46.3 ; 55.5]$ & 0.45 \\
\hline
\end{tabular}

Values are presented as median with and 1 st and 3rd quartile

$L V E D V i$ indexed left ventricular end-diastolic volume, LVESVi indexed left ventricular end-systolic volume, $L V S V I$ indexed left ventricular stroke volume, $L V E F$ left ventricular ejection fraction, $L V M M i$ indexed left ventricular myocardial mass

*Comparisons were performed using the Mann-Whitney U test. Statistically significant p values are indicated in bold

LVEF was reduced (Table 3). Median indexed left ventricular end-systolic volume (LVESVi) from CMR analysis was significantly higher in patients compared to controls whereas indexed left ventricular end-diastolic volume (LVEDVi) and indexed left ventricular myocardial mass (LVMMi) were not significantly different. There was no difference between TA and DILV patients regarding indexed LV volumes, LVEF and LVMMi, all derived by CMR (Table 3). Patients with an age $>20$ years had higher values for LVEDVi, LVESVi and LVMMi as well as a reduced LVEF (Table 4).

Median GS and GSR values from 2D-CMR-FT are shown in Table 5. Compared to healthy controls, patients had significantly reduced values for GLS and global circumferential strain (GCS) and global circumferential strain rate (GCSR). There was no difference for GLSR and global radial strain rate (GRSR) as well as for global radial strain (GRS) measured by $2 \mathrm{D}-\mathrm{CMR}-\mathrm{FT}$ between patients and controls. 2D-CMR-FT derived GLS, GCS and GRS correlated with LVEF from CMR volumetry in the entire patient group (Fig. 2). When comparing TA and DILV patients there was no statistically significant difference for GLS, GLSR, GCS, GCSR, GRS and GRSR measurements from 2D-CMR-FT between both groups. Patients $>20$ years of age had lower values for GLS, GCS, GRS, GCSR and GRSR (Table 4).

Out of the 54 patients, 44 underwent echocardiography within 3 months of the CMR study. 7 patients were excluded from the analysis due to poor image quality or inability to visualize the entire LV. Comparison between 2D-CMR-FT and 2D-STE are illustrated in Table 6 as well as in Fig. 3. Mean GLSR by 2D-CMR-FT was higher than by 2D-STE $(-1.2 \pm 0.41 / \mathrm{s}$ vs. $-0.9 \pm 0.21 / \mathrm{s}, \mathrm{p}<0.001)$. No difference was found for GLS and longitudinal strain at basal, mid and apical level. Bland-Altman-Plots are demonstrated in Fig. 4 and show that the agreement for the GLS measurements using 2D-CMR-FT and 2D-STE was acceptable.
Table 4 CMR data across age groups in SLV patients

\begin{tabular}{llll}
\hline Parameter & $<10$ years $(\mathrm{n}=23)$ & $10-20$ years $(\mathrm{n}=24)$ & $>20$ years $(\mathrm{n}=7)$ \\
\hline GLS $(\%)$ & $-15.2[-18.5 ;-13.7]$ & $-16.0[-18.7 ;-14.8]$ & $14.6[-16.7 ;-13.4]$ \\
GLSR $(1 / \mathrm{s})$ & $-1.3[-1.6 ;-1.0]$ & $-1.2[-1.4 ;-0.9]$ & $-1.2[-1.7 ;-0.8]$ \\
GCS $(\%)$ & $-20.3[-21.9 ;-17.9]$ & $-21.6[-24.3 ;-18.8]$ & $17.1[-22.0 ;-16.2]$ \\
GCSR $(1 / \mathrm{s})$ & $-1.2[-1.4 ;-1.2]$ & $-1.2[-1.3 ;-1.0]$ & $-1.1[-1.4 ;-0.9]$ \\
GRS $(\%)$ & $51.2[45.8 ; 60.8]$ & $53.1[42.4 ; 61.9]$ & $44.5[38.9 ; 62.1]$ \\
GRSR $(1 / \mathrm{s})$ & $2.5[2.2 ; 2.9]$ & $1.9[1.6 ; 2.3]$ & $1.8[1.7 ; 2.1]$ \\
LVEDVi $\left(\mathrm{ml} / \mathrm{m}^{2}\right)$ & $76.5[66.8 ; 86.6]$ & $82.5[73.9 ; 93.4]$ & $85.0[74.2 ; 104.8]$ \\
LVESVi $\left(\mathrm{ml} / \mathrm{m}^{2}\right)$ & $35.9[27.0 ; 43.6]$ & $32.1[28.1 ; 41.8]$ & $44.5[35.6 ; 53.1]$ \\
LVSVi $\left(\mathrm{ml} / \mathrm{m}^{2}\right)$ & $41.2[38.3 ; 46.2]$ & $46.7[42.0 ; 52.2]$ & $44.8[35.9 ; 53.0]$ \\
LVEF $(\%)$ & $55.3[51.5 ; 58.5]$ & $58.5[52.3 ; 62.4]$ & $51.1[43.1 ; 52.1]$ \\
LVMMi $\left(\mathrm{g} / \mathrm{m}^{2}\right)$ & $47.7[42.1 ; 55.6]$ & $51.5[43.4 ; 62.6]$ & $53.7[44.3 ; 71.9]$ \\
\hline
\end{tabular}

Values are presented as median with interquartile range

$G L S$ global longitudinal strain, GLSR global longitudinal strain rate, GCS global circumferential strain, GCSR global circumferential strain rate, GRS global radial strain, GRSR global radial strain rate, LVEDVi indexed left ventricular end-diastolic volume, LVESVi indexed left ventricular end-systolic volume, $L V S V I$ indexed left ventricular stroke volume, $L V E F$ left ventricular ejection fraction, $L V M M i$ indexed left ventricular myocardial mass 
Table 5 Comparison of 2D-CMR-FT data between patients and controls as well as between patients with TA and DILV

\begin{tabular}{lllllll}
\hline Parameter & $\begin{array}{l}\text { Single LV } \\
(\mathrm{n}=54)\end{array}$ & $\begin{array}{l}\text { Controls } \\
(\mathrm{n}=35)\end{array}$ & $\begin{array}{l}* \mathrm{p} \\
\text { value }\end{array}$ & $\begin{array}{l}\text { TA } \\
(\mathrm{n}=24)\end{array}$ & $\begin{array}{l}\text { DILV } \\
(\mathrm{n}=18)^{\dagger}\end{array}$ & $\begin{array}{l}* \mathrm{p} \\
\text { value }\end{array}$ \\
\hline GLS $(\%)$ & $-15.8[-18.3 ;-14.2]$ & $-24.1[-26.3 ;-22.5]$ & $<\mathbf{0 . 0 0 0 1}$ & $-15.3[-18.5 ;-14.2]$ & $-16.3[-17.6 ;-14.5]$ & 0.64 \\
GLSR $(1 / \mathrm{s})$ & $-1.2[-1.5 ;-1.0]$ & $-1.3[-1.4 ;-1.1]$ & 0.28 & $-1.3[-1.5 ;-1.0]$ & $-1.4[-1.7 ;-1.1]$ & 0.69 \\
GCS $(\%)$ & $-20.5[-23.3 ;-17.7]$ & $-30.2[-33.5 ;-29.0]$ & $<\mathbf{0 . 0 0 0 1}$ & $-20.0[-23.2 ;-17.2]$ & $-19.6[-23.2 ;-17.2]$ & 0.48 \\
GCSR (1/s) & $-1.2[-1.3 ;-1.1]$ & $-1.8[-2.0 ;-1.6]$ & $<\mathbf{0 . 0 0 0 1}$ & $-1.2[-1.3 ;-1.1]$ & $-1.2[-1.3 ;-1.0]$ & 0.78 \\
GRS (\%) & $51.2[42.6 ; 61.8]$ & $55.9[46.2 ; 62.4]$ & 0.47 & $49.7[44.1 ; 58.0]$ & $53.6[43.7 ; 64.7]$ & 0.46 \\
GRSR (1/s) & $2.2[1.8 ; 2.7]$ & $2.1[1.9 ; 2.6]$ & 0.90 & $2.2[1.9 ; 2.5]$ & $2.3[1.9 ; 2.5]$ & 0.67 \\
\hline
\end{tabular}

$G L S$ global longitudinal strain, GLSR global longitudinal strain rate, GCS global circumferential strain, GCSR global circumferential strain rate, GRS global radial strain, GRSR global radial strain rate

*Comparisons were performed using the Mann-Whitney U test. Statistically significant $\mathrm{p}$ values are indicated in bold

${ }^{\dagger}$ GLS- and GLSR-Average were measured in 19 patients. Values are presented as median with interquartile range
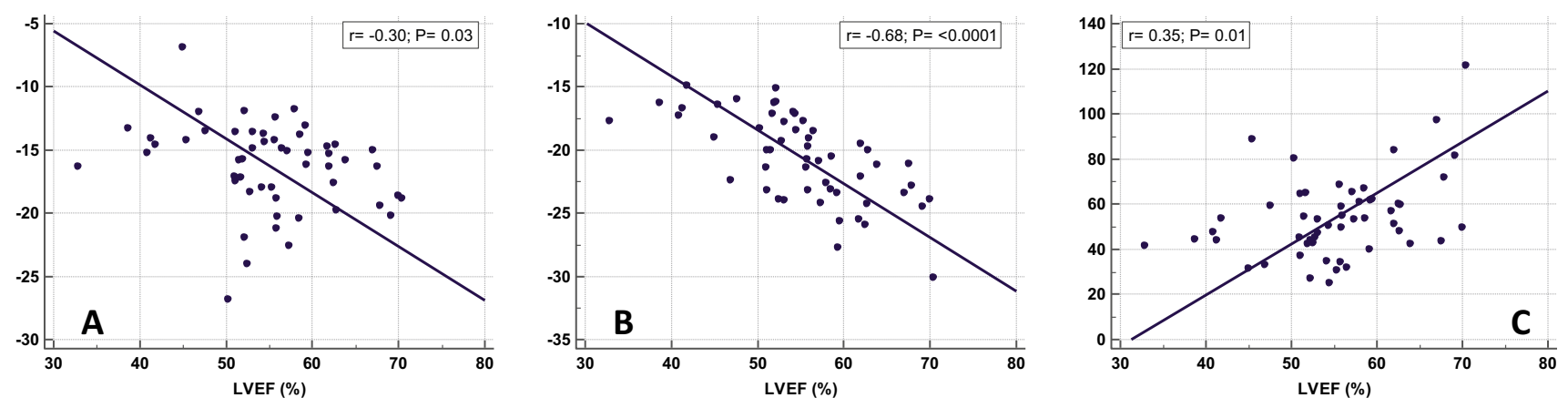

Fig. 2 Graphs showing the associations between LVEF and global longitudinal strain (a) as well as between circumferential and radial strain (b and $\mathbf{c}$ ) in the entire patient cohort

Table 6 Comparison of global and regional longitudinal deformation parameters measured by 2D-CMR-FT and 2D-STE

\begin{tabular}{lllc}
\hline Myocardial deformation & $\begin{array}{l}\text { CMR-FT } \\
(\mathrm{n}=38)\end{array}$ & $\begin{array}{l}\text { 2D-STE } \\
(\mathrm{n}=37)\end{array}$ & *p value \\
\hline GLS (\%) & $-16.7 \pm 3.2$ & $-16.3 \pm 3.9$ & 0.63 \\
GLSR (1/s) & $-1.3 \pm 0.3$ & $-0.9 \pm 0.2$ & $<\mathbf{0 . 0 0 0 1}$ \\
LS LV base (\%) & $-18.3 \pm 7.0$ & $-16.4 \pm 4.3$ & 0.16 \\
LS LV mid-cavity (\%) & $-16.5 \pm 5.5$ & $-16.5 \pm 3.4$ & 0.98 \\
LS LV apex (\%) & $-15.7 \pm 5.7$ & $-16.0 \pm 8.6$ & 0.88 \\
\hline
\end{tabular}

Statistically significant $\mathrm{p}$ values are indicated in bold

$G L S$ global longitudinal strain, GLSR global longitudinal strain rate, $L S L V$ base left ventricular longitudinal strain at the basal level, $L S$ $L V$ mid-cavity left ventricular longitudinal strain at the mid-ventricular level, $L S L V$ apex left ventricular longitudinal strain at the apex

\section{Discussion}

One of strengths of the present study is that a relatively large cohort of SLV patients $(n=54)$ were included, and that they were compared to healthy controls. Although median LVEF, GLS and GCS from 2D-CMR-FT were reduced compared to controls, most patients had a normal NYHA functional class.

\section{Myocardial deformation and function in single left ventricle patients compared to controls}

Strain analyses using 2D-CMR-FT and 2D-STE in SV patients have been performed by other groups, but most studies included small and mixed patient cohorts (17, 20) $[17,20]$. The present study, however, included a relatively large cohort of SLV patients $(n=54)$ and found significantly reduced values for GLS, GCS and GCSR by $2 \mathrm{D}-\mathrm{CMR}-\mathrm{FT}$ compared to healthy controls. $\mathrm{Hu}$ et al. observed significantly reduced GCS and GRS values in Fontan patients compared to controls using CMR [21]. Different to our study, they only included patients with a preserved LVEF (> 55\%) and concluded that global and regional circumferential strains could be used for early detection of abnormal myocardial function [21]. That strain values might be impaired before the ejection fraction $(\mathrm{EF})$ is compromised has been demonstrated also in 
Fig. 3 Example of 2D-CMR-FT (a) and 2D-STE (b) analysis in a patient with tricuspid atresia after TCPC completion

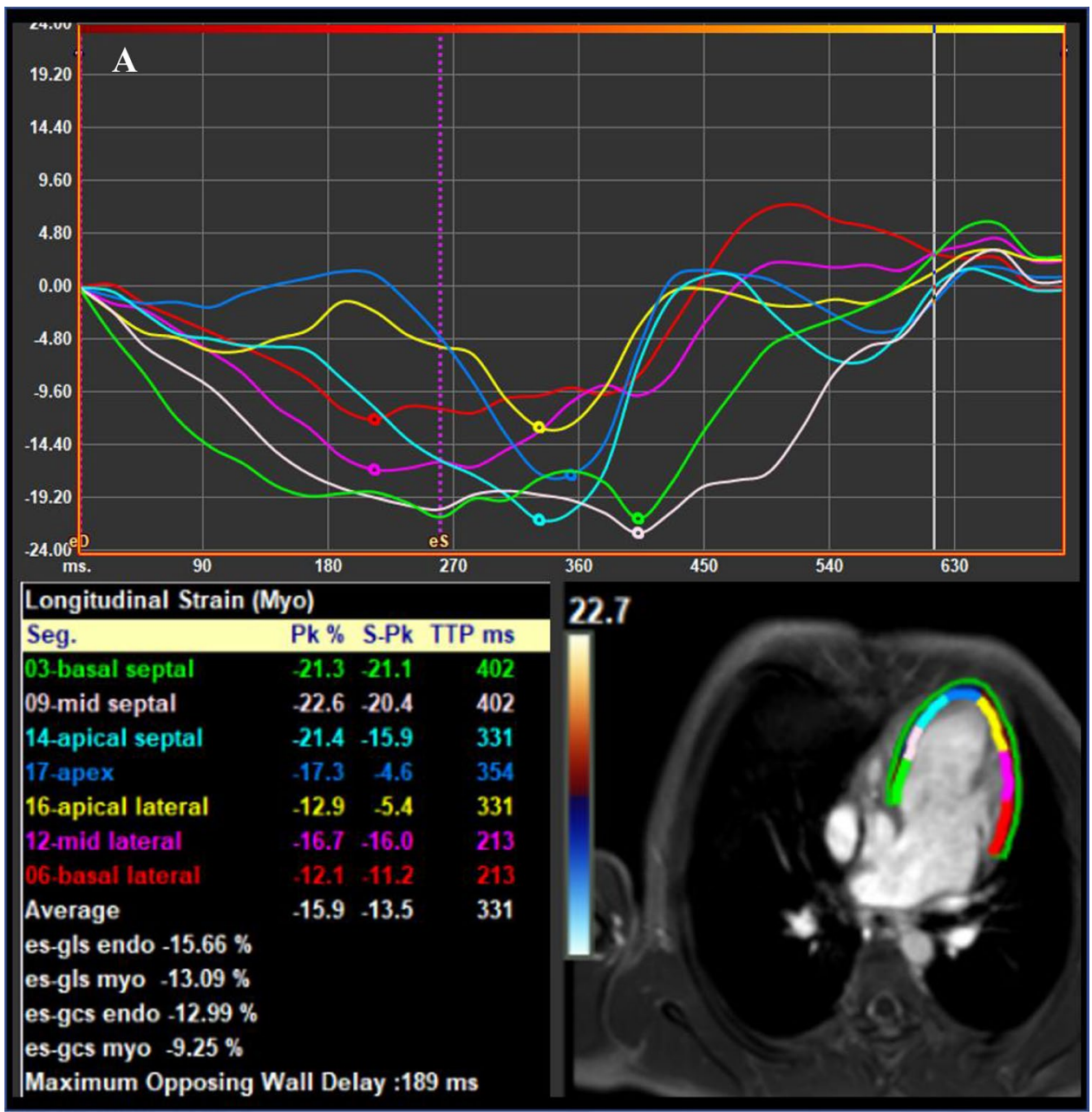

Fig. 3 (continued)

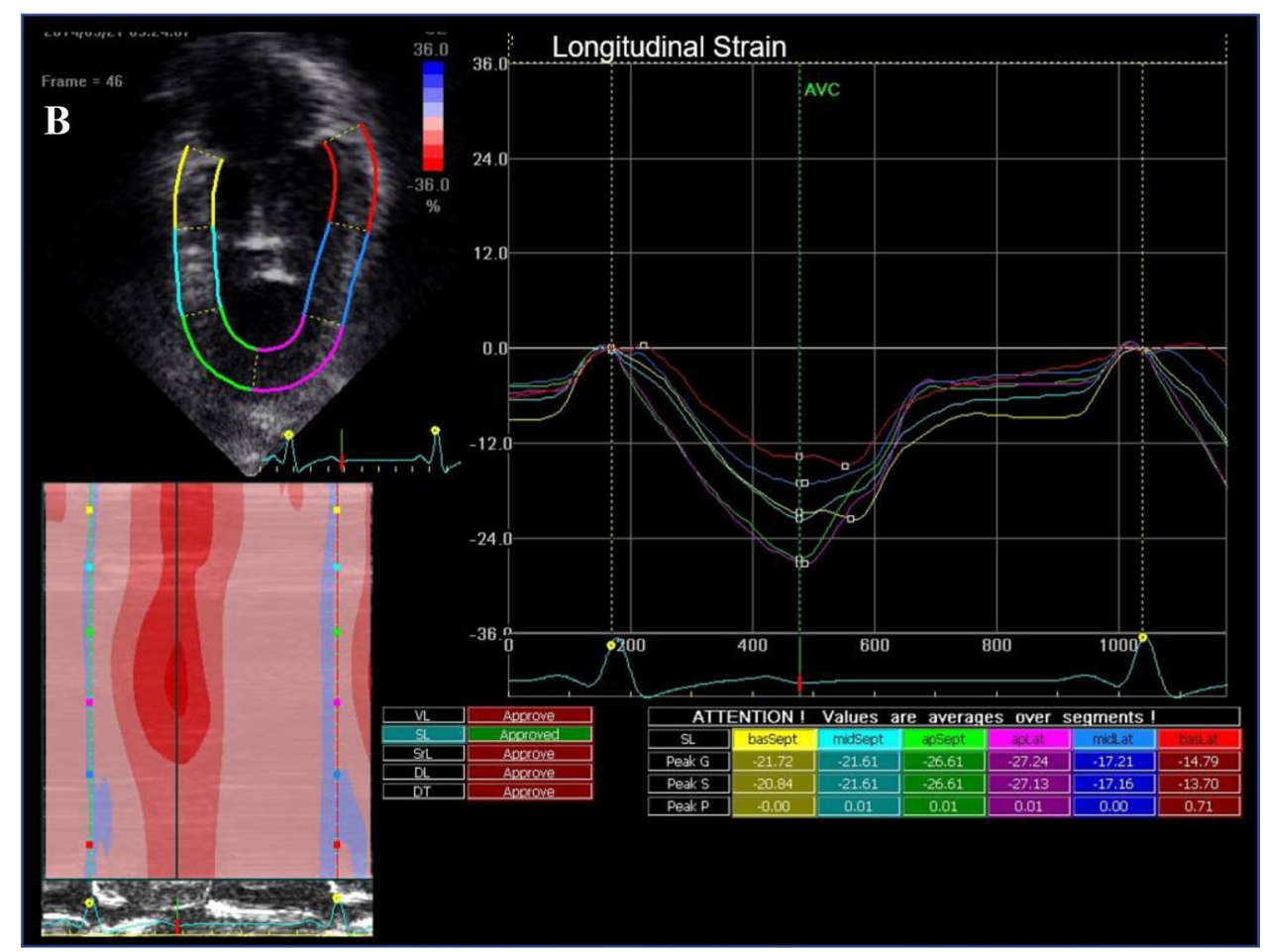


GLS

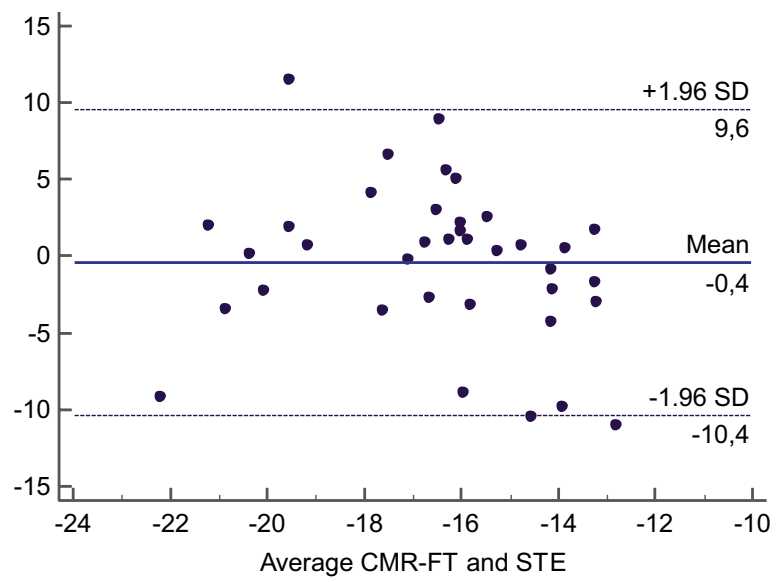

LS LV mid

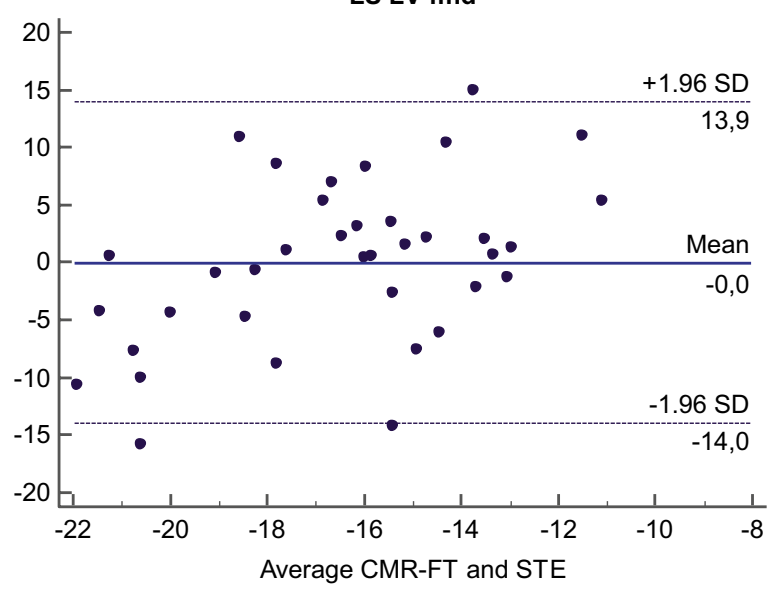

LS LV base

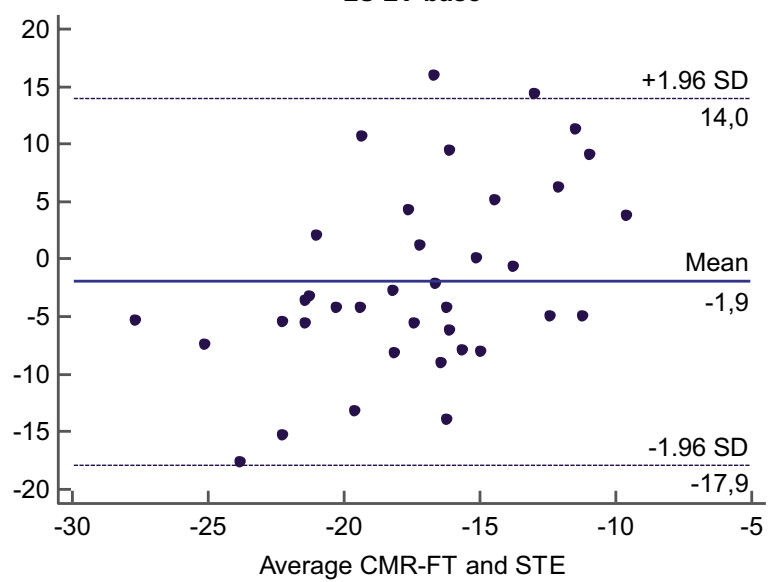

LS LV apex

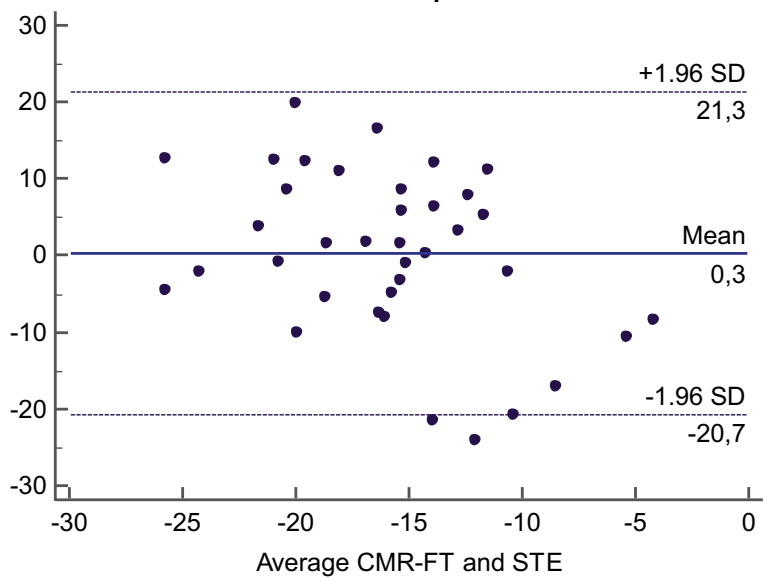

Fig. 4 Bland-Altman-Plots comparing 2D-CMR-FT and 2D-STE. Horizontal solid lines represent the mean difference between both analysis techniques and the dashed lines indicate the mean \pm 1.96 standard deviation of the difference

various other patient groups [22,23] and it has been shown that a preserved EF might be explained mathematically through geometric factors [24].

More than $50 \%$ of our SLV patients had a preserved LVEF measured by CMR volumetry but compared to controls LVEF in patients was significantly reduced. Similar findings have been reported by Singh et al. in a small $(n=16)$ SLV patient cohort [18]. They found a lower LVEF and larger volumetric indices using CMR in pediatric TA patients compared to healthy subjects [18]. Other groups found a reduced LVEF, however they also included patients with a with SRV [14]. A reduction in LVEF in SLV patients compared to controls might be explained by different hemodynamics in some patients and by a heterogeneity in myocardial function in SLV patients [18, 20]. Moreover, an abnormal myoarchitecture as reported in TA patients has to be considered [25].
We were able to show that LVEF from CMR data in SLV patients correlates with GCS, GLS and GRS measured by 2D-CMR-FT. Other groups have shown similar relationships between EF and strain values [24, 26]. Nevertheless, correlations between myocardial deformation parameters and EF are a matter of debate. Lipiec et al. suggested a non-linear hemiellipsoid model to explain the association between systolic GLS and LVEF [27]. More recently a mathematical model has been introduced describing the relationship between LVEF, GCS and GLS [28]. In this model a reduction in LVEF would correspond to reduced GCS and GLS values [28].

\section{Comparison between tricuspid atresia and double inlet left ventricle patients}

To our knowledge, no study has compared LV myocardial deformation and function in TA and DILV patients using 
CMR imaging. Our findings do not suggest any major difference in myocardial deformation, function and size between these two entities. An impaired left ventricular function in patients with TA compared to DILV was found in a cardiac catheterization study by Redington et al. Unfortunately, these results are not comparable with our data from a technical point of view (different imaging modalities) [29].

\section{Comparison between 2-dimensional cardiovascular magnetic resonance feature tracking and 2-dimensional speckle tracking echocardiography}

In our study we found clinically acceptable agreement between 2D-CMR-FT and 2D-STE, however, only 37 echocardiographic examinations could be analyzed. Schmidt et al. analyzed a mixed cohort of adult Fontan patients including both SLV and SRV patients. They highlighted the fact that 2D-CMR-FT allows analyzing all myocardial segments whereas STE is commonly limited by the acoustic windows [14]. Similarly, in our study we had to exclude seven echocardiographic studies because of poor image quality but all CMR examinations were suitable for strain analyses. A study from Ghelani et al. assessed the reproducibility of strain measurements in Fontan patients using 2D-CMR-FT and 2D-STE. Their results suggested that deformation analyses from different modalities should not be mixed [13]. Different to them we did not perform intra-modality reproducibility analyses and we are therefore unable to draw a similar conclusion. However, since 2D-CMR-FT was possible in all SLV patients compared to 2D-STE and that it has become more easily available for routine CMR analyses, we believe that 2D-CMR-FT is a good alternative to 2D-STE. Furthermore, CMR reference values for LV strain values in children and adults exist and can be used for comparison [30, 31].

\section{Study limitations}

The retrospective design of the study implies some limitations. First, in some patients certain CMR data sets were missing and were therefore not available for analysis. In addition, GLS was only measured from the 4-chamber view or axial cine images and this might have impacted our strain results.

Future studies are needed to evaluate the fate of the LV in SLV patients during follow up.

The number of healthy controls was smaller, but since both groups were age-matched this could not influence the study findings.

Finally, we did not perform an intermodality reproducibility analysis for CMR-FT and 2D-STE.

\section{Conclusions}

Most SLV patients had a normal NYHA functional class and $52 \%$ of patients had a preserved, CMR derived, LVEF $(\geq 55 \%)$. However, compared to controls, LVEF, GLS, GCS and GCSR measured by CMR were reduced. Our results suggest that LV deformation and function in SLV patients may behave differently compared to a normal LV in healthy subjects. Follow up studies evaluating the fate of the LV in SLV patients are needed. 2D-CMR-FT might be a suitable modality in this setting.

Acknowledgements This study was supported by Deutsches Zentrum für Herz-Kreislaufforschung e.V. We also thank Mrs Traudel Hansen for her support with patient management.

Funding Open Access funding enabled and organized by Projekt DEAL.

Availability of data and materials All data and materials support the published claims and comply with field standards.

\section{Declarations}

Conflict of interest The authors declare that they have no conflict of interest.

Consent to participate Informed consent was obtained from all individual participants included in the study.

Consent for publication All authors have given their consent for publication.

Ethical approval All procedures performed in studies involving human participants were in accordance with the ethical standards of the institutional research committee (ethics committee of the medical faculty of the Christian-Albrechts University Kiel, No. D555/19) and with the 1964 Helsinki declaration and its later amendments or comparable ethical standards.

Open Access This article is licensed under a Creative Commons Attribution 4.0 International License, which permits use, sharing, adaptation, distribution and reproduction in any medium or format, as long as you give appropriate credit to the original author(s) and the source, provide a link to the Creative Commons licence, and indicate if changes were made. The images or other third party material in this article are included in the article's Creative Commons licence, unless indicated otherwise in a credit line to the material. If material is not included in the article's Creative Commons licence and your intended use is not permitted by statutory regulation or exceeds the permitted use, you will need to obtain permission directly from the copyright holder. To view a copy of this licence, visit http://creativecommons.org/licenses/by/4.0/.

\section{References}

1. Schwartz I, McCracken CE, Petit CJ, Sachdeva R (2018) Late outcomes after the Fontan procedure in patients with single ventricle: 
a meta-analysis. Heart 104:1508-1514. https://doi.org/10.1136/ heartjnl-2017-312807

2. Poh CL, d'Udekem Y (2018) Life after surviving Fontan surgery: a meta-analysis of the incidence and predictors of late death. Heart Lung Circ 27:552-559. https://doi.org/10.1016/j.hlc.2017.11.007

3. d'Udekem Y, Iyengar AJ, Galati JC, Forsdick V, Weintraub RG, Wheaton GR, Bullock A, Justo RN, Grigg LE, Sholler GF, Hope S, Radford DJ, Gentles TL, Celermajer DS, Winlaw DS (2014) Redefining expectations of long-term survival after the Fontan procedure: twenty-five years of follow-up from the entire population of Australia and New Zealand. Circulation 130:S32-S38. https://doi.org/10.1161/CIRCULATIONAHA.113.007764

4. de Leval MR, Deanfield JE (2010) Four decades of Fontan palliation. Nat Rev Cardiol 7:520-527. https://doi.org/10.1038/nrcar dio.2010.99

5. Rychik J, Atz AM, Celermajer DS, Deal BJ, Gatzoulis MA, Gewillig MH, Hsia TY, Hsu DT, Kovacs AH, McCrindle BW, Newburger JW, Pike NA, Rodefeld M, Rosenthal DN, Schumacher KR, Marino BS, Stout K, Veldtman G, Younoszai AK, d'Udekem Y (2019) Evaluation and management of the child and adult with Fontan circulation: a scientific statement from the American Heart Association [published online ahead of print, $2019 \mathrm{Jul} 1$ 1]. Circulation. https://doi.org/10.1161/CIR.0000000000000696

6. Khairy P, Fernandes SM, Mayer JE Jr, Triedman JK, Walsh EP, Lock JE, Landzberg MJ (2008) Long-term survival, modes of death, and predictors of mortality in patients with Fontan surgery. Circulation 117:85-92. https://doi.org/10.1161/CIRCULATIO NAHA.107.738559

7. Atz AM, Zak V, Mahony L, Uzark K, D'agincourt N, Goldberg DJ, Williams RV, Breitbart RE, Colan SD, Burns KM, Margossian R, Henderson HT, Korsin R, Marino BS, Daniels K, McCrindle BW, Pediatric Heart Network Investigators (2017) Longitudinal outcomes of patients with single ventricle after the Fontan procedure. J Am Coll Cardiol 69:2735-2744. https://doi.org/10.1016/j. jacc.2017.03.582

8. Fogel MA, Weinberg PM, Chin AJ, Fellows KE, Hoffman EA (1996) Late ventricular geometry and performance changes of functional single ventricle throughout staged Fontan reconstruction assessed by magnetic resonance imaging. J Am Coll Cardiol 28:212-221. https://doi.org/10.1016/0735-1097(96)00111-8

9. Kato A, Riesenkampff E, Yim D, Yoo SJ, Seed M, Grosse-Wortmann L (2017) Pediatric Fontan patients are at risk for myocardial fibrotic remodeling and dysfunction. Int J Cardiol 240:172-177. https://doi.org/10.1016/j.ijcard.2017.04.073

10. Rathod RH, Prakash A, Powell AJ, Geva T (2010) Myocardial fibrosis identified by cardiac magnetic resonance late gadolinium enhancement is associated with adverse ventricular mechanics and ventricular tachycardia late after Fontan operation. J Am Coll Cardiol 55:1721-1728. https://doi.org/10.1016/j.jacc.2009.12.036

11. Kutty S, Rathod RH, Danford DA, Celermajer DS (2016) Role of imaging in the evaluation of single ventricle with the Fontan palliation. Heart 102:174-183. https://doi.org/10.1136/heart jnl-2015-308298

12. Ginde S, Goot BH, Frommelt PC (2017) Imaging adult patients with Fontan circulation. Curr Opin Cardiol 32:521-528. https:// doi.org/10.1097/HCO.0000000000000422

13. Ghelani SJ, Harrild DM, Gauvreau K, Geva T, Rathod RH (2016) Echocardiography and magnetic resonance imaging based strain analysis of functional single ventricles: a study of intra- and intermodality reproducibility. Int J Cardiovasc Imaging 32:1113-1120

14. Schmidt R, Orwat S, Kempny A, Schuler P, Radke R, Kahr PC, Hellige A, Baumgartner H, Diller GP (2014) Value of speckletracking echocardiography and MRI based feature tracking analysis in adult patients after Fontan-type palliation. Congenit Heart Dis 9:397-406. https://doi.org/10.1111/chd.12156
15. Salehi Ravesh M, Rickers C, Bannert FJ, Hautemann D, Al Bulushi A, Gabbert DD, Wegner P, Kis E, Hansen JH, JeroschHerold MM, Kramer HH, Logoteta J (2018) Longitudinal deformation of the right ventricle in hypoplastic left heart syndrome: a comparative study of 2D-feature tracking magnetic resonance imaging and 2D-speckle tracking echocardiography. Pediatr Cardiol 39:1265-1275. https://doi.org/10.1007/s00246-018-1892-x

16. Ho PK, Lai CT, Wong SJ, Cheung YF (2012) Three-dimensional mechanical dyssynchrony and myocardial deformation of the left ventricle in patients with tricuspid atresia after Fontan procedure. J Am Soc Echocardiogr 25:393-400. https://doi.org/10.1016/j. echo.2012.01.003

17. Moiduddin N, Texter KM, Zaidi AN, Hershenson JA, Stefaniak C, Hayes J, Cua CL (2010) Two-dimensional speckle strain and dyssynchrony in single left ventricles vs. normal left ventricles. Congenit Heart Dis 5:579-586. https://doi.org/10.1111/j.17470803.2010.00460.x

18. Singh GK, Cupps B, Pasque M, Woodard PK, Holland MR, Ludomirsky A (2010) Accuracy and reproducibility of strain by speckle tracking in pediatric subjects with normal heart and single ventricular physiology: a two-dimensional speckle-tracking echocardiography and magnetic resonance imaging correlative study. J Am Soc Echocardiogr 23:1143-1152. https://doi.org/10.1016/j. echo.2010.08.010

19. Cerqueira MD, Weissman NJ, Dilsizian V, Jacobs AK, Kaul S, Laskey WK, Pennell DJ, Rumberger JA, Ryan T, Verani MS, American Heart Association Writing Group on Myocardial Segmentation and Registration for Cardiac Imaging (2002) Standardized myocardial segmentation and nomenclature for tomographic imaging of the heart. A statement for healthcare professionals from the Cardiac Imaging Committee of the Council on Clinical Cardiology of the American Heart Association. Int J Cardiovasc Imaging 18:539-542

20. Lunze FI, Lunze K, McElhinney DB, Colan SD, Gauvreau K, Lange PE, Schmitt B, Berger F (2013) Heterogeneity of regional function and relation to ventricular morphology in patients with Fontan circulation. Am J Cardiol 112:1207-1213. https://doi.org/ 10.1016/j.amjcard.2013.06.016

21. Hu L, Sun A, Guo C, Ouyang R, Wang Q, Yao X, Zhong Y (2019) Assessment of global and regional strain left ventricular in patients with preserved ejection fraction after Fontan operation using a tissue tracking technique. Int J Cardiovasc Imaging 35:153-160. https://doi.org/10.1007/s10554-018-1440-z

22. Berganza FM, de Alba CG, Özcelik N, Adebo D (2017) Cardiac magnetic resonance feature tracking biventricular two-dimensional and three-dimensional strains to evaluate ventricular function in children after repaired tetralogy of Fallot as compared with healthy children. Pediatr Cardiol 38:566-574. https://doi.org/10. 1007/s00246-016-1549-6

23. Kutty S, Rangamani S, Venkataraman J, Li L, Schuster A, Fletcher SE, Danford DA, Beerbaum P (2013) Reduced global longitudinal and radial strain with normal left ventricular ejection fraction late after effective repair of aortic coarctation: a CMR feature tracking study. Int J Cardiovasc Imaging 29:141-150. https://doi.org/10. 1007/s10554-012-0061-1

24. Stokke TM, Hasselberg NE, Smedsrud MK, Sarvari SI, Haugaa KH, Smiseth OA, Edvardsen T, Remme EW (2017) Geometry as a confounder when assessing ventricular systolic function: comparison between ejection fraction and strain. J Am Coll Cardiol 70:942-954. https://doi.org/10.1016/j.jacc.2017.06.046

25. Sanchez-Quintana D, Climent V, Ho SY, Anderson RH (1999) Myoarchitecture and connective tissue in hearts with tricuspid atresia. Heart 81:182-191. https://doi.org/10.1136/hrt.81.2.182

26. Altman M, Bergerot C, Aussoleil A, Davidsen ES, Sibellas F, Ovize M, Bonnefoy-Cudraz E, Thibault H, Derumeaux G (2014) 
Assessment of left ventricular systolic function by deformation imaging derived from speckle tracking: a comparison between 2D and 3D echo modalities. Eur Heart J Cardiovasc Imaging 15:316-323. https://doi.org/10.1093/ehjci/jet103

27. Lipiec P, Wiśniewski J, Kasprzak JD (2014) Should we search for linear correlations between global strain parameters and ejection fraction? Eur Heart J Cardiovasc Imaging 15:1301. https://doi.org/ 10.1093/ehjci/jeu020

28. Pedrizzetti G, Lapinskas T, Tonti G, Stoiber L, Zaliunas R, Gebker R, Pieske B, Kelle S (2019) The relationship between EF and strain permits a more accurate assessment of LV systolic function. JACC Cardiovasc Imaging 12:1893-1895. https://doi.org/ 10.1016/j.jcmg.2019.03.019

29. Redington AN, Knight B, Oldershaw PJ, Shinebourne EA, Rigby ML (1988) Left ventricular function in double inlet left ventricle before the Fontan operation: comparison with tricuspid atresia. Br Heart J 60:324-331. https://doi.org/10.1136/hrt.60.4.324

30. Kawel-Boehm N, Hetzel SJ, Ambale-Venkatesh B, Captur G, Francois CJ, Jerosch-Herold M, Salerno M, Teague SD,
Valsangiacomo-Buechel E, van der Geest RJ, Bluemke DA (2020) Reference ranges ("normal values") for cardiovascular magnetic resonance (CMR) in adults and children: 2020 update. J Cardiovasc Magn Reason 22:87. https://doi.org/10.1186/ s12968-020-00683-3

31. André F, Robbers-Visser D, Helling-Bakki A, Föll A, Voss A, Katus HA, Helbing WA, Buss SJ, Eichhorn JG (2016) Quantification of myocardial deformation in children by cardiovascular magnetic resonance feature tracking: determination of reference values for left ventricular strain and strain rate. J Cardiovasc Magn Reson. https://doi.org/10.1186/s12968-016-0310-x

Publisher's Note Springer Nature remains neutral with regard to jurisdictional claims in published maps and institutional affiliations. 